ApJL ACCEPTED

Preprint typeset using $\mathrm{LATEX}_{\mathrm{E}}$ style emulateapj v. 11/10/09

\title{
SHORT LIFETIME OF PROTOPLANETARY DISKS IN LOW-METALLICITY ENVIRONMENTS ${ }^{1}$
}

\author{
Chikako Yasui $^{2,3}$, NaOto Kobayashi ${ }^{3}$, Alan T. Tokunaga ${ }^{4}$, Masao Saito $^{2}$, and Chiniro Tokoku ${ }^{5}$ \\ ApJL accepted
}

\begin{abstract}
We studied near-infrared disk fractions of six young clusters in the low-metallicity environments with $[\mathrm{O} / \mathrm{H}] \sim-0.7$ using deep $J H K$ images with Subaru $8.2 \mathrm{~m}$ telescope. We found that disk fraction of the low-metallicity clusters declines rapidly in $<1 \mathrm{Myr}$, which is much faster than the $\sim 5-$ 7 Myr observed for the solar-metallicity clusters, suggesting that disk lifetime shortens with decreasing metallicity possibly with an $\sim 10^{Z}$ dependence. Since the shorter disk lifetime reduces the time available for planet formation, this could be one of the major reasons for the strong planet-metallicity correlation. Although more quantitative observational and theoretical assessments are necessary, our results present the first direct observational evidence that can contribute to explaining the planetmetallicity correlation.

Subject headings: Galaxy: abundances - infrared: stars - open clusters and associations: general - planetary systems: protoplanetary disks - stars: formation - stars: pre-main sequence
\end{abstract}

\section{INTRODUCTION}

Because planets are formed in protoplanetary disks, the lifetime of protoplanetary disks $\left(\tau_{\text {disk }}\right)$ is thought to be one of the most fundamental parameters directly connected to the planet formation probability $\left(P_{\mathrm{pl}}\right)$. In particular, in the current standard core-accretion scenario of gas giant planet formation (Safronov 1969; Lissauer \& Stevenson 2007), a long disk lifetime of more than $10 \mathrm{Myr}$ was thought to be necessary to produce the planetesimals that eventually become the rocky core of the giant planets (e.g., $100 \mathrm{Myr}$; Lissauer 1993). However, Strom et al. (1989) studied the time variation of the frequency of disk-harboring stars in Taurus molecular cloud that have $K$-band excess and suggested that the disk lifetime is in the range from $\ll 3 \mathrm{Myr}$ to $\sim 10 \mathrm{Myr}$, which is much shorter than was thought to be necessary. After their pioneering work, a notion called the "disk fraction", which is defined as the frequency of near-infrared (NIR) to mid-infrared (MIR) excess stars (disk-harboring stars) within a young cluster, was developed and widely used to study the disk lifetime (e.g., Lada 1999; Haisch et al. 2001; Hernández et al. 2007). In all studies, the disk fraction is found to decrease as a function of cluster age, showing that the disk lifetime is about 5-7 Myr for nearby young clusters. Because the observation with NIR/MIR is sensitive to only heated dust located at the inner disk with a stellocentric distance of $\sim 0.1$ to a few AU, there still remained a possibility that the disk lifetime derived with NIR/MIR excess emission is only for the limited region of the disk. However, from the (sub-)millimeter continuum observa-

\footnotetext{
${ }^{1}$ Based on data collected at Subaru Telescope, which is operated by the National Astronomical Observatory of Japan.

2 National Astronomical Observatory of Japan 2-21-1 Osawa, Mitaka, Tokyo, 181-8588, Japan: ck.yasui@nao.ac.jp

${ }^{3}$ Institute of Astronomy, School of Science, University of Tokyo, 2-21-1 Osawa, Mitaka, Tokyo 181-0015, Japan

${ }^{4}$ Institute for Astronomy, University of Hawaii, 2680 Woodlawn Drive, Honolulu, HI 96822, USA

${ }^{5}$ Astronomical Institute, Tohoku University, Aramaki, Aoba, Sendai 980-8578, Japan
}

tion of cold dust $(\sim 10 \mathrm{~K})$ in the outer disk ( $\gtrsim 50 \mathrm{AU})$ by Andrews \& Williams (2005), the lifetime of the outer disk is also found to be similar, thus the entire disk is now thought to disappear nearly simultaneously. After the discovery that $\tau_{\text {disk }}$ is less than $10 \mathrm{Myr}$, it is widely accepted that giant planet cores must form in this short time for any theoretical models (Lissauer \& Stevenson 2007).

Although as many as over 450 exoplanets are now known (Schneider et al. 2009), these planets are unexpectedly found to have a lot of diversity in terms of e.g., mass, orbital period, and eccentricity. Perhaps the most telling discovery in exoplanet study is the "planetmetallicity correlation", the higher probability of a star hosting a giant planet with increasing metallicity (e.g., Fischer et al. 2005), suggesting that metallicity could be the most crucial parameter for giant planet formation. Although this correlation is generally interpreted as a natural consequence of the core-accretion scenario, the origin of it is still not well-explained despite detailed analyses by either theoretical studies assuming solar metallicity or observations of solar metallicity regions (e.g., Ida \& Lin 2004b; Wyatt et al. 2007). Therefore, it is necessary to directly study protoplanetary disks under different metallicity conditions to find any clues of the planet-metallicity correlation and to test theories of it.

Since our Galaxy is known to have a metallicity gradient with lower metallicity for larger Galactic radius, ranging from $\simeq-1$ dex to $\simeq+0.5$ dex (e.g., Rudolph et al. 2006), we can explore different metallicity regions at large distances with high-sensitivity observations with large telescopes. As a first step, we focused on the lowmetallicity environment in the outer region of our Galaxy $\left(R_{g} \gtrsim 15 \mathrm{kpc}\right)$ to study a region with significantly lower metallicity $([\mathrm{O} / \mathrm{H}] \sim-1 \mathrm{dex})$. As a first result, we derived the disk fraction of two clusters in Digel Cloud 2 at $R_{g}=19 \mathrm{kpc}$ (Yasui et al. 2009, hereafter Paper I), which are low metallicity $([\mathrm{O} / \mathrm{H}] \simeq-0.7)$ and very young $(\sim 0.5 \mathrm{Myr}$ old $)$ clusters. Disk fractions for both clusters were found to be quite low in spite of their very young 
age, suggesting that the disk lifetime in low metallicity is short. In this paper, we summarize the disk lifetime in low-metallicity environments with our all targets in the outer Galaxy and discuss the implication for the planetmetallicity correlation.

\section{TARGET SELECTION AND OBSERVATION}

We searched the literature for star-forming regions in the outer Galaxy and with $[\mathrm{O} / \mathrm{H}]<-0.5$. Among the listed 10 candidate star-forming regions, we selected four regions at $R_{g} \gtrsim 15 \mathrm{kpc}$ and in the second quadrant of the Galaxy as an initial set of samples, mostly by considering the visibility from the Northern hemisphere. We also checked whether the candidate regions have at least one associated stellar aggregate using Two Micron All Sky Survey (2MASS) data. As a result, we selected six clusters (Table 1): one cluster in each Sh 2-207 and Sh 2-208 (Bica et al. 2003), and two in each Sh 2-209 (Klein et al. 2005) and Digel Cloud 2 (Kobayashi et al. 2008). The average metallicity of all target clusters is $\simeq-0.7 \mathrm{dex}$, which is significantly lower than the solar metallicity.

Deep $J H K_{S}$-band images of the target star-forming regions were obtained with the $8.2 \mathrm{~m}$ Subaru telescope equipped with MOIRCS (Multi-Object InfraRed Camera and Spectrograph; Suzuki et al. 2008), which has a wide field of view $\left(4^{\prime} \times 7^{\prime}\right)$ and uses the MKO NIR photometric filters (Tokunaga et al. 2002). The weather condition was photometric with excellent seeing $(\simeq 0$.' $3-0$ !' 4$)$ for the Cloud 2 and Sh 2-209 observations on 2006 September 3 UT, while a little cirrus was present with seeing of $\sim 1^{\prime \prime}$ for the Sh 2-207 and Sh 2-208 observations on 2006 November 8, 2007 November 23, and 2008 January 14 UT. Total integration time for each wavelength band was $\sim 500-1000$ sec. After the raw data were reduced with standard IRAF procedures, $J H K$ aperture photometry was performed. For most clusters aperture photometry with IRAF apphot was enough even for such large distances because stellar images are sufficiently smaller than the stellar separations. Only for two dense clusters in Sh 2-209, we performed photometry with point-spread function fitting using IRAF daophot with scripts for automated photometry, "autodao" 6 .

Photometric standard stars that were obtained at the similar airmass as the object fields are used except for Sh 2-207 and Sh 2-208 clusters, for which 2MASS stars in the fields are used after converting the 2MASS magnitudes to the MKO magnitudes with the color transformations in Leggett et al. (2006). The achieved limiting magnitudes $(10 \sigma), J \simeq 20-22 \mathrm{mag}, H \simeq 19-21 \mathrm{mag}$, and $K_{S} \simeq 18.5-21 \mathrm{mag}$, correspond to $\lesssim 0.1 M_{\odot}$. This mass detection limit, sufficiently less than $1 M_{\odot}$, enables a direct comparison of disk fractions in such distant clusters with those of star-forming clusters in the solar neighborhood (cf. Haisch et al. 2001). For each embedded cluster, we identified cluster members in the same way as for the Cloud 2 clusters $($ Paper I $)$ : the extinction criterion $\left(A_{V}\right)$ for identifying cluster members was determined for each cluster considering the $A_{V}$ distributions in the cluster region and the field region (see details in Paper I). The details for each cluster will be published in separated papers. 6 Copyright (C) 2008-2009 Noriyuki Matsunaga

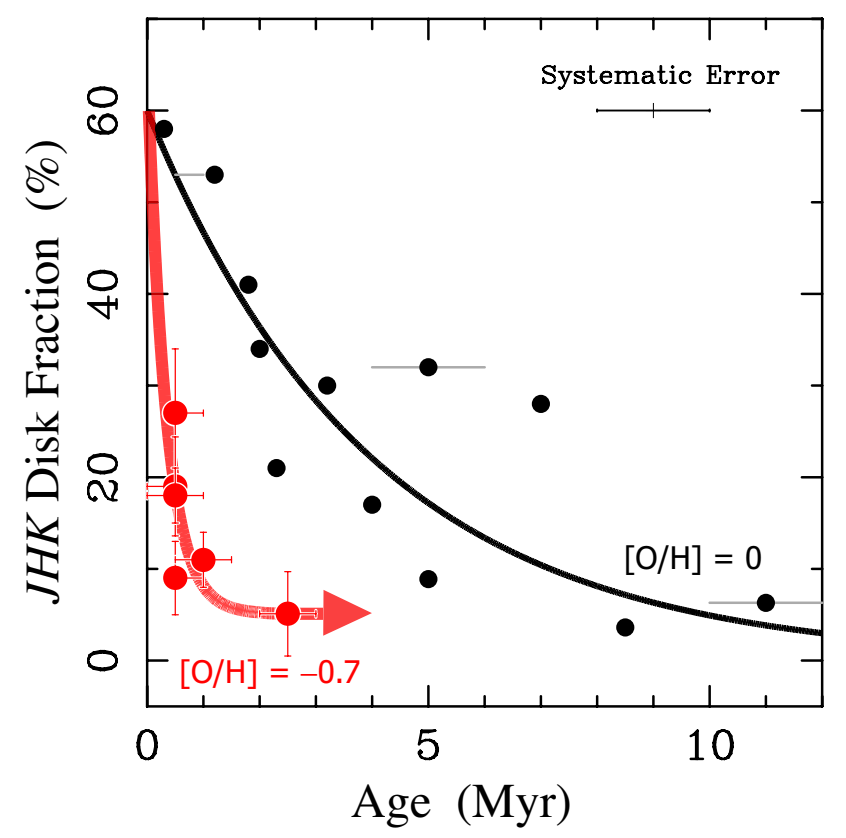

FIG. 1.- Disk fraction as a function of cluster age. JHK disk fractions of the young clusters with low metallicity are shown by red filled circles, while those of young clusters with solar metallicity are shown by black filled circles. The typical systematic error of ages for the solar metallicity cluster is shown at the top-right corner (see the main text for explanation of the gray horizontal lines for three of the clusters). The black line shows the disk fraction evolution under solar metallicity, while the red arrow shows the proposed $J H K$ disk fraction evolution in low-metallicity environments. Note that these lines are drawn by eye and are not derived with rigorous fitting.

\section{DERIVATION OF AGE AND DISK FRACTION}

\subsection{Clusters in Low Metallicity}

Following the method by Muench et al. (2000), we first derived the ages of clusters by comparing observed the $K$-band luminosity function (KLF) with model KLFs of various ages constructed from the canonical initial mass function (IMF), mass-luminosity $(M-L)$ relation and distance of the cluster. The detail of the model fitting is described in Paper I in the case of Cloud 2. We applied the same procedure to all clusters. We confirmed that the variation of the IMF and $M-L$ relation in the relevant metallicity range is small enough so that the ages of the clusters can be estimated within the uncertainty of $\lesssim 1$ Myr (Yasui et al. 2006), which is sufficient for the present study. Estimated ages of most targets are found to be very young, 1 Myr, but one cluster, Sh 2-207, shows a relatively old age $(2-3 \mathrm{Myr})$, as expected from the 2MASS image, which shows a less-extincted cluster.

Next, we used $J H K$ color-color diagrams to derive the disk fractions for each cluster. The method we used to determine cluster infrared excess fractions from colorcolor diagrams is described in detail in Paper I along with extensive discussions on the uncertainties. In Table 1 , we list the derived disk fractions and ages for the entire sample clusters, and plotted the results in Figure 1. The uncertainties of the disk fraction reflect Poisson errors. Only for the Sh 2-207 cluster we derived the disk fraction by considering the contamination from the field stars because it is difficult to identify cluster members with tadko hatpall extinction of this older cluster. The number 
TABLE 1

JHK DISK FRACTIONS OF EMBEDDED CLUSTERS IN LOW-METALLICITY ENVIRONMENTS.

\begin{tabular}{llcll}
\hline \hline \multicolumn{1}{c}{ Cluster } & $\begin{array}{c}\log [\mathrm{O} / \mathrm{H}]^{a} \\
(\mathrm{dex})\end{array}$ & $\begin{array}{c}R_{g} / D^{b} \\
(\mathrm{kpc})\end{array}$ & $\begin{array}{c}\text { Age } \\
(\mathrm{Myr})\end{array}$ & \multicolumn{1}{c}{$\begin{array}{c}\text { Disk Fraction } \\
(\%)\end{array}$} \\
\hline Cloud 2-N & $-0.7(1)$ & $19 / 12(\mathrm{i})$ & $0.5-1$ & $9 \pm 4(5 / 52)$ \\
Cloud 2-S & $-0.7(1)$ & $19 / 12(\mathrm{i})$ & $0.5-1$ & $27 \pm 7(16 / 59)$ \\
Sh 2-207 & $-0.7(2)$ & $12 / 4^{d}(\mathrm{ii})$ & $2-3$ & $5.1 \pm 4.6(1.2 / 23.4)^{e}$ \\
Sh 2-208 & $-0.8(2)$ & $12 / 4^{d}(\mathrm{ii})$ & 0.5 & $19 \pm 5.5(12 / 63)$ \\
Sh 2-209 $\operatorname{main}^{f}$ & $-0.6(2)$ & $17.5 / 10$ (iii) & $0.5-1$ & $10 \pm 0.8(163 / 1605)$ \\
Sh 2-209 $\operatorname{sub}^{f}$ & $-0.6(2)$ & $17.5 / 10(\mathrm{iii})$ & $0.5-1$ & $7.1 \pm 1.2(35 / 494)$ \\
\hline
\end{tabular}

References. - (1) Lubowich et al. (2004), (2) Caplan et al. (2000); (i) Kobavashi et al. (2008), (ii) Fich et al. (1990), (iii) Chini \& Wink (1984).

Note. - ${ }^{a}$ Metallicity of the observed regions. The solar metallicity is assumed as $\log (\mathrm{O} / \mathrm{H})_{\odot}=8.7($ Asplund et al. 2009$)$. ${ }^{b}$ Adopted distance $(D)$ and Galactic distance $\left(R_{g}\right)$. We assumed the Galactic distance of the sun as $R_{\odot}=8.0 \mathrm{kpc}($ Reid 1993$)$. ${ }^{c}$ Mass detection limit of the data. ${ }^{d}$ For both Sh $2-207$ and Sh $2-208$ clusters, we adopted the kinematic distances because model KLFs with the photometric distances $(D \simeq 8-9$ kpc, $\left.R_{g} \simeq 15.5-16.5 \mathrm{kpc}\right)$ do not match the observed KLF at all, while those with the kinematic distances $(D \simeq 4 \mathrm{kpc})$ match very well. ${ }^{e}$ See detail in the main text. ${ }^{f}$ Sh 2-209 has two clusters, which are located near the two peaks of millimeter continuum (Klein et al. 2005): hereafter we call the larger cluster at the northeast side and the smaller cluster at the southwest side as "main cluster" and "sub-cluster", respectively.

TABLE 2

JHK DISK FRACTIONS OF EMBEDDED CLUSTERS WITH AGES OF $>5$ MYR IN THE SOLAR NEIGHBORHOOD.

\begin{tabular}{llllll}
\hline \hline Cluster & $\begin{array}{l}\text { Age }^{a} \\
(\mathrm{Myr})\end{array}$ & $\begin{array}{l}\text { Disk Fraction } \\
(\%)\end{array}$ & $\begin{array}{l}M_{\text {lim }^{b}} \\
\left(M_{\odot}\right)\end{array}$ & Filter $^{c}$ & Ref $^{d}$ \\
\hline Orion OB1b & $5(1)$ & $8.9 \pm 3$ & 0.1 & 2 MASS & 1 \\
Upper Sco & $5(2)$ & $32 \pm 4$ & 0.1 & 2MASS & 3 \\
$\eta$ Cham & $6(4)$ & $28 \pm 12$ & 0.08 & 2MASS & 5 \\
Orion OB1a & $8.5(1)$ & $3.6 \pm 2.5$ & 0.1 & 2MASS & 1 \\
NGC 7160 & $10(5)$ & $6.3 \pm 3.6$ & 0.4 & 2MASS & 6 \\
\hline
\end{tabular}

Note. — ${ }^{a}$ References for the ages are shown in the parenthesis. Although there are two age estimates for Orion OB1b and OB1a by using different isochrone models, we show the average value here. ${ }^{b}$ Mass detection limit of the data. ${ }^{c}$ The photometric system of the obtained $J H K$ data. ${ }^{d}$ References for the $J H K$ photometric data. For Upper Scorpius and NGC 7160, we used JHK magnitudes in 2MASS All-Sky Point Source Catalog for the stars listed in the references.

References. - (1) Briceño et al. (2005), (2) Preibisch et al. (2002), (3) Carpenter et al. (2006), (4) Luhman \& Steeghs (2004), (5) Sicilia-Aguilar et al. (2005), (6) Sicilia-Aguilar et al. (2006).

of stars in the Sh 2-207 cluster region is 38 and of these only 2 have disks. The normalized number of stars in the field region is estimated to be $14.6 \pm 0.9$ with that lying in the infrared excess region of the diagrams to be $0.8 \pm 0.2$. Therefore, the final disk fraction of the Sh 2-207 cluster is estimated at $5.1 \% \pm 4.6 \%((2-0.8) /(38-14.6))$.

\subsection{Reference Clusters in Solar Metallicity}

For comparison, we also derived the $J H K$ disk fraction and age of various embedded clusters in the solar neighborhood in a uniform way using the photometry data in the literature. We chose publications with the following criteria: (1) JHK photometry data set of cluster members in the same filter system are available for a reliable estimate and (2) the mass detection limit is $\leq 1 M_{\odot}$ (Haisch et al. 2001) to sufficiently cover the most typical mass range in order to have the most representative disk fraction for each cluster. In addition to seven young $(<5 \mathrm{Myr})$ clusters in the solar neighborhood used in Paper I (see Table 21), we derived disk fractions for additional five clusters with older ages ( $\geq 5 \mathrm{Myr}$; Orion OB1b, Upper Scorpius, $\eta$ Cham, Orion OB1a, and NGC
7160; see Table 2).

Although the ages of these clusters are derived with other methods than KLF fitting (e.g., with spectroscopy and imaging data using the H-R diagram), we derived the ages of the clusters with KLF fitting for better consistency. Because the KLF fitting is reliable only for good signal-to-noise imaging data which cover the peak magnitude of KLF, we derived ages of Trapezium, Upper Sco, and NGC 7160 clusters, since these are the only clusters for which we could find such data in the literature. The resultant ages are identical to those in the literature in spite of larger uncertainties with increasing ages as shown with thick gray horizontal lines in Figure 1. Considering that ages of reference clusters estimated with various pre-main-sequence models have systematic uncertainty of $\sim 1$ Myr (Haisch et al. 2001), the age estimate of clusters with low-metallicity with KLF fitting in $\S 3.1$ appears to be reliable.

For all clusters, the $J H K$ disk fractions were estimated in the same way as for the clusters in low-metallicity environments. The resultant disk fractions and ages for solar-metallicity clusters are shown in Figure 1 with black circles. They show decreasing disk fraction with increasing age: the decline is rapid up to $5-7 \mathrm{Myr}$ and stays almost flat beyond this point with disk fraction slowly declining from $10 \%$ to $5 \%$. These characteristics are totally identical to the latest MIR disk fractions based on ground-based $L$-band observations and Spitzer observations (see Figure 14 in Hernández et al. 2007).

\section{DISK LIFETIME IN LOW-METALLICITY ENVIRONMENTS}

Figure 1 clearly shows that the disk fractions of all the clusters in low-metallicity environments are less than $30 \%$, which is significantly lower than nearby embedded clusters of similar age. Also, the disk fraction with low metallicity seems to reach the first lowest level $(\sim 10 \%)$ at the age of $\sim 1 \mathrm{Myr}$, which corresponds to the disk fraction with solar metallicity at $\sim 5-7 \mathrm{Myr}$. In combination with the fact that there are no embedded clusters of solar metallicity whose disk fractions are this low at the same ages, our results show that disk fraction strongly depends on metallicity, confirming our earlier suggestion in Paper I.

Following the detailed discussion in Paper I, our re- 
sults suggest that the entire disk dispersal occurs in the very early phase in low-metallicity environments, while initially (at age $=0 \mathrm{Myr}$ ) an inner disk as well as outer disk exists even under low metallicity. The disk lifetime in low metallicity is estimated at $\sim 1$ Myr from Figure 1, while that in solar metallicity is $\sim 5-7 \mathrm{Myr}$, suggesting that very short disk lifetime in low-metallicity environment. Assuming that the disk lifetime--metallicity relation is described as $\tau_{\text {disk }} \propto 10^{a Z}$, the constant $a$ is estimated to be $1 \pm 0.5$ despite that we have only two measurement points ( 0 dex and $-0.7 \mathrm{dex}$ ) at present. The uncertainty of the constant comes from the uncertainties of metallicity and age of each cluster. Note that our NIR observations are sensitive to only heated dust in the inner disk (see detail in $\S 1$ ) and millimeter/sub-millimeter observations of the outer disk (e.g., with ALMA) in the near future are very important to conclude that the above estimated disk lifetime is actually for the entire disk.

The metallicity dependence of the disk lifetime may impose constraints on the mechanism of disk dispersal, which is still under debate even for solar metallicity environments. Among five major mechanisms of disk dispersal: planet formation, stellar encounter, stripping by winds, mass accretion, and photoevaporation (Hollenbach et al. 2000), only photoevaporation by the radiation of the central star appears to cause the strong metallicity dependence (see discussion in Paper I; Ercolano \& Clarke 2010) if the dominant radiation is in far-UV (FUV, $6 \mathrm{eV}<h \nu<13.6 \mathrm{eV}$; e.g., Gorti \& Hollenbach 2009a) and/or X-rays as suggested in Paper I ( $h \nu>0.1 \mathrm{keV}$; e.g., Ercolano \& Clarke 2010). However, because the theory of photoevaporation is still under development and with numerous parameters, further studies are needed. The metallicity dependencies of disk lifetime from theories are $\propto 10^{0.3 Z}$ and $\propto 10^{0.5 Z}$ for FUV photoevaporation from the theories of Gorti \& Hollenbach (2009a) and X-ray photoevaporation from those of Ercolano \& Clarke (2010), respectively. This is not enough to explain our results, although it is marginally consistent within the uncertainty $\left(10^{a Z}\right.$, $a=1 \pm 0.5)$. Note that something specific to the outer Galaxy environment (e.g., Haywood 2008) might be the reason for the rapid disk dispersal. However, considering that Sh 2-207 and Sh 2-208 clusters, which are not found to be located in the outer Galaxy region $\left(R_{g}=12 \mathrm{kpc}\right.$, see note in Table 1), also show low disk fraction and thus short disk lifetime, metallicity could be the dominant factor.

\section{IMPLICATION TO THE PLANET-METALLICITY CORRELATION}

The observed planet-metallicity correlation is known to have strong metallicity dependence $\left(P_{\mathrm{pl}} \propto 10^{2 Z}\right.$; Fischer et al. 2005). Although the core-accretion model qualitatively explains the dependence, e.g., $P_{\mathrm{pl}} \propto 10^{Z}$ in the case of Ida \& Lin (2004b), which is a deterministic model based on the core accretion, it is still not enough to explain the steep $10^{2 Z}$ dependence (see e.g., Wvatt et al. 2007). The short disk lifetime under low-metallicity suggests that planet formation becomes more difficult with decreasing metallicity, and therefore it may explain a part of the strong planet-metallicity correlation. In Paper I, we proposed that by adding the contribution from the disk lifetime, most of the observed metallicity dependence of the planet formation probability could be reasonably explained. Recently, Ercolano \& Clarke (2010) also estimated the correlation between $P_{\mathrm{pl}}$ and $\tau_{\text {disk }}$ by combining their photoevaporation model and the core-accretion model by Ida \& Lin (2004b) to suggest that the metallicity dependence of photoevaporation only plays a secondary role with an effect of $\lesssim 10^{0.2 Z}$ on the planet formation probability. However, there are more ambiguities and complexities in the planet formation process than the theoretical planet formation scenarios now available (e.g., Ida \& Lin 2004a). Also, the derived metallicity dependence of the disk lifetime still has a large uncertainty (see $\S$ 4), and needs to be confirmed by observations of clusters with higher than solar metallicity. The metallicity dependence of the disk lifetime suggested here is the only direct observational evidence for the metallicity dependence of the planet formation and could be an important clue to understanding the planet formation mechanism.

C.Y. has been supported by the Japan Society for the Promotion of Science (JSPS).

\section{REFERENCES}

Andrews, S. M., \& Williams, J. P. 2005, ApJ, 631, 1134

Asplund, M., Grevesse, N., Sauval, A. J., \& Scott, P. 2009, ARA\&A, 47, 481

Bica, E., Dutra, C. M., Soares, J., \& Barbuy, B. 2003, A\&A, 404, 223

Briceño, C., Calvet, N., Hernández, J., Vivas, A. K., Hartmann, L., Downes, J. J., \& Berlind, P. 2005, AJ, 129, 907

Caplan, J., Deharveng, L., Peña, M., Costero, R., \& Blondel, C. 2000, MNRAS, 311, 317

Carpenter, J. M., Mamajek, E. E., Hillenbrand, L. A., \& Meyer, M. R. 2006, ApJ, 651, L49

Chini, R., \& Wink, J. E. 1984, A\&A, 139, L5

Ercolano, B., \& Clarke, C. J. 2010, MNRAS, 402, 2735

Fich, M., Dahl, G. P., \& Treffers, R. R. 1990, AJ, 99, 622

Fischer, Debra A., \& Valenti, J. 2005, ApJ, 622, 1102

Gorti, U., \& Hollenbach, D. 2009, ApJ, 690, 1539

Haisch, K. E., Jr., Lada, E. A., \& Lada, C. J. 2001, ApJ, 553, L153

Haywood, M. 2008, A\&A, 482, 673

Hernández, J., et al. 2007, ApJ, 662, 1067
Hollenbach, D. J., Yorke, H. W., \& Johnstone, D. 2000, in Protostars and Planets IV, ed. V. Mannings, A. P. Boss, \& S. S. Russell (Tucson, AZ: Univ. Arizona Press), 401

Ida, S., \& Lin, D. N. C. 2004a, ApJ, 604, 388

Ida, S., \& Lin, D. N. C. 2004b, ApJ, 616, 567

Klein, R., Posselt, B., Schreyer, K., Forbrich, J., \& Henning, T. 2005, ApJS, 161, 361

Kobayashi, N., Yasui, C., Tokunaga, A. T., \& Saito, M. 2008, ApJ, 683, 178

Lada, E. A. 1999, in The Origin of Stars and Planetary Systems, ed. C. J. Lada \& N. D. Kylafis (Dordrecht: Kluwer), 441

Leggett, S. K., et al. 2006, MNRAS, 373, 781

Lissauer, J. J. 1993, ARA\&A, 31, 129

Lissauer, J. J., \& Stevenson, D. J. 2007, in Protostars and Planets V, ed. B. Reipurth, D. Jewitt, \& K. Keil (Tucson, AZ: Univ. Arizona Press), 591

Lubowich, D. A., Brammer, G., Roberts, H.,Millar, T. J., Henkel, C., \& Pasachoff, J. M. 2004, in Origin and Evolution of the

Elements, ed. A. McWilliam, \& M. Rauch (Carnegie Observ. 4; Cambridge: Cambridge Univ. Press), 37

Luhman, K. L., \& Steeghs, D. 2004, ApJ, 609, 917 
Muench, A. A., Lada, E. A., \& Lada, C. J. 2000, ApJ, 533, 358

Preibisch, T., Brown, A. G. A., Bridges, T., Guenther, E., \& Zinnecker, H. 2002, AJ, 124, 404

Reid, M. J. 1993, ARA\&A, 31, 345

Rudolph, A. L., Fich, M., Bell, G. R., Norsen, T., Simpson, J. P., Haas, M. R., \& Erickson, E. F. 2006, ApJS, 162, 346

Safronov, V. S. 1969, Evolution of the Protoplanetary Cloud and Formation of the Earth and the Planets [1972 English translation: NASA TTF-677] (Moscow: Nauka Press)

Schneider J. 2009. Extrasolar Planets Encyclopaedia.

http://exoplanet.eu/catalog-all.php

Sicilia-Aguilar, A., Hartmann, L. W., Hernández, J., Briceño, C., \& Calvet, N. 2005, AJ, 130, 188

Sicilia-Aguilar, A., et al. 2006, ApJ, 638, 897
Strom, K. M., Strom, S. E., Edwards, S., Cabrit, S., \& Skrutskie, M. F. 1989, AJ, 97, 1451

Suzuki, R., et al. 2008, PASJ, 60, 1347

Tokunaga, A. T., Simons, D. A., \& Vacca, W. D. 2002, PASP, 114,180

Wyatt, M. C., Clarke, C. J., \& Greaves, J. S. 2007, MNRAS, 380, 1737

Yasui, C., Kobayashi, N., Tokunaga, A. T., Terada, H., \& Saito, M. 2006, ApJ, 649, 753

Yasui, C., Kobayashi, N., Tokunaga, A. T., Saito, M., \& Tokoku, C. 2009, ApJ, 705, 54 (Paper I) 